\title{
Investigation and Analysis on the Management Standard of Blood Glucose Monitoring for Clinical Nurses in Nyingchi Region of Tibet
}

\author{
Haihua Zou ${ }^{1}$, Lihong Liu' ${ }^{2}$, Lijuan Dong ${ }^{2}$, Tefan Zhang ${ }^{2}$, Peiru Zhou ${ }^{{ }^{*}}$ \\ ${ }^{1}$ Nursing Department, First Affiliated Hospital of Jinan University, Guangzhou, China \\ ${ }^{2}$ Linzhi People's Hospital, Linzhi, China \\ Email:313761095@qq.com, *luzhmm@126.com
}

How to cite this paper: Zou, H.H., Liu, L.H., Dong, L.J., Zhang, T.F. and Zhou, P.R. (2020) Investigation and Analysis on the Management Standard of Blood Glucose Monitoring for Clinical Nurses in Nyingchi Region of Tibet. Open Journal of Preventive Medicine, 10, 108-119.

https://doi.org/10.4236/ojpm.2020.106008

Received: May 7, 2020

Accepted: June 9, 2020

Published: June 12, 2020

Copyright $\odot 2020$ by author(s) and Scientific Research Publishing Inc. This work is licensed under the Creative Commons Attribution International License (CC BY 4.0).

http://creativecommons.org/licenses/by/4.0/

(c) (i) Open Access

\begin{abstract}
Background: In participating in the medical support work in the Nyingchi area of Tibet, the author found that the medical education resources in this area are scarce, and the clinical nurses in this area have relatively insufficient knowledge about blood glucose monitoring and management. Accurately understanding the knowledge-attitude/belief-practice (KAP) level of nurses in Nyingchi area on blood glucose management is of great significance to the development of blood glucose monitoring management guidance for nurses in this area. Therefore, this study investigated the current status of the management of blood glucose monitoring management standards for clinical nurses in Nyingchi, Tibet. Objective: To investigate and analyze the current situation of clinical nurses in Nyingchi region of Tibet to grasp the regulation of blood glucose monitoring and guide the implementation of the norm of blood glucose monitoring for clinical nurses. Methods: The research survey was carried out from March to April 2020. Random sampling method was used to select 134 clinical in-service nurses in Nyingchii, Tibet. The self-designed knowledge-attitude/belief-practice survey questionnaire for clinical nurses' blood glucose monitoring and management in Tibet was used. Nurses' blood glucose monitoring management "KAP" scores and related influencing factors are analyzed. Results: Blood sugar monitoring and management knowledge score is $(36.78 \pm 6.80)$, attitude dimension is $(42.48 \pm 6.42)$, practice dimension is $(61.87 \pm 10.24)$ and total score is $(304.73 \pm 36.24)$. The nurse's department and whether to participate in diabetes training are the main influencing factors of the blood glucose monitoring management norms, knowledge and behavior (both $\mathrm{P}<0.05$ ). Conclusion: The standard knowledge of blood glucose monitoring and management of clinical nurses in Nyingchi region of Tibet is at the middle level, positive attitude and unsatis-
\end{abstract}


factory behavior. Nursing managers should conduct standardized training for clinical nurses in blood glucose monitoring, develop the training mode of diabetes specialty nursing talents in Nyingchi area, and construct a complete management system of diabetes specialty nursing.

\section{Keywords}

Tibet Region, Clinical Nurse, Blood Glucose Monitoring,

Knowledge-Attitude/Belief-Practice

\section{Introduction}

Diabetes is a group of metabolic diseases characterized by chronic high blood glucose and carbohydrate, fat and protein metabolic disorders, which are caused by defective insulin secretion and/or insulin action [1]. At present, there are 92.4 million adults with diabetes and 148 million people with pre-diabetes in China [2]. The prevalence of adult diabetes in China has soared from $0.67 \%$ in 1980 to $10.4 \%$ in 2013, and among the 25 leading causes of death in 2017, diabetes-related causes ranked eighth, up 11 from 1990. As a result of the high-calorie, high-protein, high-salt, high-fat and high-sugar diet, most residents have the habit of drinking alcohol for a long time [3]. The above factors lead to an increasing trend of diabetes prevalence in the region [4]. At present, the management and operation of blood glucose monitoring are mainly carried out by nurses, and the degree of nurses' mastery of blood glucose monitoring is related to the blood glucose control level and the quality of life of patients [5]. During my participation in Tibet's medical aid work in Nyingchi region, I found that due to the special geographical location of Tibet, its economic conditions are relatively backward compared with other regions, medical and educational resources are also relatively scarce, and the knowledge of blood glucose monitoring and management of clinical nurses in this region is relatively lacking. In order to accurately understand the level of knowledge, credit, and execution about blood glucose management among nurses in Nyingchi area, we analyze the influencing factors of blood glucose monitoring and management behavior of nurses, and do a good job in training and guiding the blood glucose management of nurses in this area. This study investigated and analyzed the current situation of clinical nurses in Nyingchi area, Tibet, in mastering the blood glucose monitoring management standard.

\section{Objects and Methods}

\subsection{Object}

Random sampling was used to select 134 clinical in-service nurses from a third-level hospital, two second-level hospitals, and two first-level hospitals (a total of 6 hospitals) in Nyingchi, Tibet, as the research object. Inclusion criteria: 1) in-service clinical or nursing management post nurses in Linzhi region; 2) 
nurses with one year or more clinical work experience; 3) agreed and volunteered to participate in this survey; 4) The sample size of each hospital is $\geq 20$ person-times. Exclusion criteria: a) nurses who were not in the post during the investigation; b) nurses who were unwilling or otherwise unwilling to participate in this investigation.

\subsection{Methodology}

\subsection{1. "Knowledge-Attitude/Belief-Practice" Theory}

Knowledge-attitude/belief-practice (KABP/KAP) is generally abbreviated as KAP, which includes abbreviations of Knowledge, Attitude, and Practice. KAP theory believes that people's changes in health behaviors are not realized in a short time, but through Long-term accumulation of health knowledge prompts people's cognitive attitudes to gradually change, which in turn contributes to the formation of people's health behaviors. Taking KAP theory as the theoretical framework for nurses' blood glucose monitoring management norms, nurses actively learn about blood glucose monitoring management related knowledge, pay attention to the factors that may affect clinical blood glucose monitoring in nursing work, and form a positive and correct attitude to blood glucose monitoring management. Through firm beliefs, the nurses can guide their patients to monitor and manage their blood glucose in their nursing work, correct inappropriate nursing operations in time, and form a reasonable blood glucose monitoring and management behavior.

\subsubsection{Survey Tools}

Based on the opinions of experts from Guangdong province and Tibet region, the questionnaire on blood glucose monitoring and management of clinical nurses in Nyingchi region of Tibet was formulated. The questionnaire mainly includes four parts: 1) the general information of the respondents, including the department, hospital level, position, professional title, education, working years, whether or not to participate in diabetes specialist training, etc. 2) - 4) is the current status of KAP of clinical nurses on blood glucose monitoring. 2) The mastery of clinical nurses' knowledge of blood glucose monitoring management in Nyingchi, Tibet, including the meaning, method, purpose and precautions of sugar monitoring knowledge, a total of 11 items. 3) The mastery of the Attitude dimension of clinical nurses' blood glucose monitoring and management in Nyingchi, Tibet, including personal abilities and personal wishes, a total of 10 items. 4) Mastery of clinical nurses' blood glucose monitoring management practice dimension in Nyingchi, Tibet, including the methods and applications of blood glucose monitoring management, a total of 16 items. This questionnaire is based on the American Diabetes Association Guidelines (2018 Edition) [6]. On the basis of this study, we consult the library materials, internet and electronic literature databases (including the databases of China Zhiwang, Weipu, PubMed, MEDLINE and Webofscience, etc.) and the related key words such as "clinical nurses, blood glucose monitoring management, information and prac- 
tice investigation theory, questionnaire design".

The questionnaire was designed after consulting and discussing the literature. According to the opinions of two diabetes nursing experts, two Tibet regional nursing experts and one endocrine medical expert in Guangdong province, Knowledge-attitude/belief-practice questionnaire of clinical nurses' blood glucose monitoring and management in Nyingchi, Tibet. The overall Cronbach's $\alpha$ of the questionnaire was 0.951 , of which the Cronbach's $\alpha$ of 11 entries in knowledge was 0.957 , the Cronbach's $\alpha$ of 10 entries in attitude was 0.968 , and the Cronbach's $\alpha$ of 16 entries in behavior was 0.914 . Entry assignment was high to low (5 - 1). The total score of knowledge, attitude and behavior dimensions was 55,50 and 80 , respectively. The higher the score of single dimension was, the higher the nurses' knowledge cognition, attitude and behavior mastery of blood glucose monitoring and management was.

\subsubsection{Survey Methodology}

The on-the-job nurses in Nyingchi region of Tibet were investigated by questionnaire star questionnaire, which restricted a mobile terminal to fill out only one questionnaire once to avoid repeated investigation. The questionnaire was completed by secret ballot, and the questionnaire was collected within $5 \mathrm{~d}$ from the date of issue. A total of 148 questionnaires were distributed, 134 valid questionnaires were recovered, and the effective recovery rate of the questionnaire was $90.5 \%$.

\subsubsection{Statistical Methods}

The survey data were analyzed by SPSSAU20.0 statistical software, the measurement data were expressed as $(\chi \pm s)$, the data comparison was carried out by chi-square test and single factor ANOVA; the counting data were expressed as the number of cases and percentage (\%), and the multivariate linear stepwise regression analysis and other statistical methods were used to $\mathrm{P} \chi \pm \mathrm{s} 0.05$ or 0.01 to indicate that the data difference was statistically significant.

\section{Results}

1) A total of 134 nurses were included in the general situation study of the subjects, 120 in tertiary hospitals and 6 in secondary hospitals, and 8 in primary hospitals, 18 of whom had attended specialized training in diabetes and 116 had not attended specialized training in diabetes, as detailed in Table 1.

2) Comparison of KAP scores of blood glucose monitoring and management of clinical nurses in Nyingchi, Tibet with different characteristics. There are no statistically significant differences in the scores of knowledge and behavior of nurses in different departments, hospitals, positions, titles, education, working years and whether they participate in diabetes specialty training, blood sugar monitoring and management knowledge cognitive dimension score (36.78 \pm $6.80)$, belief dimension score (42.48 \pm 6.42$)$, behavior dimension score (61.87 \pm $10.24)$ and total score $(304.73 \pm 36.24)$, the nurse's knowledge, belief, behavior 
dimension score in terms of the nurse's department, hospital level, nurse position, nurse title, working years There are significant differences $(P<0.05)$, see Table 2 for details.

Table 1. General information on survey subjects $(\mathrm{N}=134)$.

\begin{tabular}{|c|c|c|c|c|c|}
\hline Projects & Classification & Count/percentage & Projects & Classification & Count/percentage \\
\hline \multirow{7}{*}{ Departments } & Internal Medicine & $34(25.37 \%)$ & Title & Nurse & $46(34.33 \%)$ \\
\hline & Surgery & $10(7.46 \%)$ & & Nurse & $70(52.24 \%)$ \\
\hline & Obstetrics and gynaecology & $16(11.94 \%)$ & & Head Nurse & $18(13.43 \%)$ \\
\hline & Pediatrics & $12(8.96 \%)$ & Education & Technical secondary & $4(2.99 \%)$ \\
\hline & Department of Intensive Medicine & $2(1.49 \%)$ & & College & $74(55.22 \%)$ \\
\hline & Emergency department & $12(8.96 \%)$ & & Undergraduate & $56(41.79 \%)$ \\
\hline & Other & $48(35.82 \%)$ & & Graduate and above & $0(0 \%)$ \\
\hline \multirow[t]{3}{*}{ Hospital level } & Level I hospitals & $8(5.97 \%)$ & Duration of service (a) & - & $2(1,5)$ \\
\hline & Level II hospitals & $6(4.48 \%)$ & Are you trained in diabetes & Yes & $18(13.43)$ \\
\hline & Level III hospitals & $120(89.55 \%)$ & & No & $116(86.57)$ \\
\hline \multirow[t]{4}{*}{ Positions } & Nurse & $98(73.13 \%)$ & & & \\
\hline & Nursing team & $12(8.96 \%)$ & & & \\
\hline & Head of Section & $18(13.43 \%)$ & & & \\
\hline & Nursing Offr/Deputy Director & $6(4.48 \%)$ & & & \\
\hline
\end{tabular}

Table 2. Comparison of KAP scores of blood glucose monitoring management norms for 134 active nurses in Nyingchi, Tibet (N =134).

\begin{tabular}{|c|c|c|c|c|c|c|c|c|c|c|}
\hline \multirow{2}{*}{ Projects } & \multirow{2}{*}{ Number/\% } & \multicolumn{3}{|c|}{ Cognitive status } & \multicolumn{3}{|c|}{ Attitude } & \multicolumn{3}{|c|}{ Conduct } \\
\hline & & Score $(\chi \pm S)$ & $x^{2}$ & $\mathbf{P}$ & Score $(\chi \pm S)$ & $x^{2}$ & $\mathbf{P}$ & Score $(\chi \pm S)$ & $x^{2}$ & $\mathbf{P}$ \\
\hline Total & 134 & $36.78 \pm 6.80$ & & & $42.48 \pm 6.42$ & & & $61.87 \pm 10.24$ & & \\
\hline Departments & & & 186.859 & 0.000 & & 141.909 & 0.000 & & 441.611 & 0.000 \\
\hline Pediatrics & $48(35.82)$ & $38.67 \pm 7.28$ & & & $43.00 \pm 7.48$ & & & $60.83 \pm 12.11$ & & \\
\hline Other & $34(25.37)$ & $36.46 \pm 7.88$ & & & $41.54 \pm 6.08$ & & & $62.04 \pm 10.77$ & & \\
\hline Internal Medicine & $10(7.46)$ & $38.53 \pm 6.53$ & & & $45.82 \pm 4.31$ & & & $64.18 \pm 9.76$ & & \\
\hline Surgery & $16(11.94)$ & $36.00 \pm 4.62$ & & & $44.20 \pm 5.83$ & & & $59.80 \pm 6.20$ & & \\
\hline Obstetrics and gynaecology & $12(8.96)$ & $33.25 \pm 5.46$ & & & $41.75 \pm 6.04$ & & & $62.13 \pm 9.16$ & & \\
\hline Emergency department & $2(1.49)$ & $37.17 \pm 4.49$ & & & $37.83 \pm 7.54$ & & & $58.00 \pm 12.20$ & & \\
\hline $\begin{array}{c}\text { Department of Intensive } \\
\text { Medicine }\end{array}$ & $12(8.96)$ & $33.00 \pm 0.00$ & & & $30.00 \pm 0.00$ & & & $56.00 \pm 0.00$ & & \\
\hline Hospital level & & & 118.615 & 0.000 & & 67.047 & 0.000 & & 146.283 & 0.000 \\
\hline Level I hospitals & $8(5.97)$ & $42.00 \pm 5.71$ & & & $40.25 \pm 0.46$ & & & $60.00 \pm 7.41$ & & \\
\hline Level II hospitals & $6(4.48)$ & $26.33 \pm 6.83$ & & & $39.00 \pm 0.89$ & & & $59.00 \pm 13.42$ & & \\
\hline Level III hospitals & $120(89.55)$ & $36.95 \pm 6.36$ & & & $42.80 \pm 6.71$ & & & $62.13 \pm 10.27$ & & \\
\hline Positions & & & 171.158 & 0.000 & & 115.918 & 0.000 & & 227.819 & 0.000 \\
\hline
\end{tabular}


Continued

\begin{tabular}{|c|c|c|c|c|c|c|c|c|c|c|}
\hline Nurse & $98(73.13)$ & $37.69 \pm 6.86$ & & & $42.22 \pm 6.59$ & & & $61.98 \pm 10.11$ & & \\
\hline Nursing Offr/Deputy Director & $6(4.48)$ & $27.67 \pm 8.50$ & & & $41.67 \pm 4.93$ & & & $61.67 \pm 14.04$ & & \\
\hline Nursing team & $12(8.96)$ & $38.17 \pm 5.02$ & & & $47.17 \pm 4.02$ & & & $62.50 \pm 10.32$ & & \\
\hline Head of Section & $18(13.43)$ & $33.89 \pm 3.38$ & & & $41.00 \pm 6.21$ & & & $60.89 \pm 10.41$ & & \\
\hline Title & & & 79.216 & 0.000 & & 58.241 & 0.000 & & 127.557 & 0.000 \\
\hline Head Nurse & $18(13.43)$ & $33.22 \pm 3.14$ & & & $41.22 \pm 6.07$ & & & $64.44 \pm 10.26$ & & \\
\hline Nurse & $46(34.33)$ & $37.61 \pm 6.83$ & & & $41.96 \pm 7.13$ & & & $61.96 \pm 9.89$ & & \\
\hline Nurse & $70(52.24)$ & $37.14 \pm 7.24$ & & & $43.14 \pm 6.02$ & & & $61.14 \pm 10.49$ & & \\
\hline Education & & & 68.431 & 0.003 & & 31.725 & 0.202 & & 139.741 & 0.000 \\
\hline Technical secondary & $4(2.99)$ & $32.00 \pm 1.15$ & & & $40.00 \pm 11.55$ & & & $53.50 \pm 13.28$ & & \\
\hline College & $74(55.22)$ & $36.92 \pm 6.45$ & & & $42.59 \pm 7.06$ & & & $62.89 \pm 10.60$ & & \\
\hline Undergraduate & $56(41.79)$ & $36.93 \pm 7.41$ & & & $42.50 \pm 5.08$ & & & $61.11 \pm 9.35$ & & \\
\hline Number of years worked & & & 189.695 & 0.000 & & 93.283 & 0.000 & & 247.466 & 0.000 \\
\hline $1-5$ years & $52(38.81)$ & $36.85 \pm 6.67$ & & & $41.31 \pm 6.97$ & & & $59.81 \pm 9.28$ & & \\
\hline $6-10$ years & $42(31.34)$ & $37.33 \pm 8.27$ & & & $44.38 \pm 5.52$ & & & $64.62 \pm 11.32$ & & \\
\hline $11-20$ years & $26(19.40)$ & $37.23 \pm 5.69$ & & & $43.31 \pm 4.91$ & & & $62.46 \pm 7.22$ & & \\
\hline $21-30$ years & $12(8.96)$ & $33.17 \pm 2.86$ & & & $37.83 \pm 6.99$ & & & $62.00 \pm 13.84$ & & \\
\hline 31 years and above & $2(1.49)$ & $39.00 \pm 0.00$ & & & $50.00 \pm 0.00$ & & & $49.00 \pm 0.00$ & & \\
\hline $\begin{array}{l}\text { Have you received diabetes } \\
\text { specialist training }\end{array}$ & & & 37.015 & 0.012 & & 19.908 & 0.098 & & 57.368 & 0.002 \\
\hline Yes & $18(13.43)$ & $38.00 \pm 5.70$ & & & $45.67 \pm 4.99$ & & & $63.89 \pm 3.89$ & & \\
\hline No & $116(86.57)$ & $36.59 \pm 6.96$ & & & $41.98 \pm 6.49$ & & & $61.55 \pm 10.87$ & & \\
\hline
\end{tabular}

3) The five items with the lowest blood sugar monitoring knowledge, attitude, behavior score and score. Clinical nurses had a minimum score of $60.29 \%$ for blood glucose monitoring knowledge dimension items, and nurses had a low knowledge level of blood glucose monitoring; clinical nurses had a minimum score of $82.08 \%$ for blood glucose monitoring attitude dimension items. Nurses attached importance to blood glucose monitoring; clinical nurses focused on blood glucose monitoring. The lowest score of the monitoring behavior dimension item is $64.77 \%$, and the nurse's behavior on blood glucose monitoring is relatively lacking; see Table 3 for details.

4) Multivariate analysis of KAP scores of blood glucose monitoring of clinical nurses in Nyingchi, Tibet. The KAP questionnaire related to blood glucose monitoring was divided into dependent variables, and the variables with statistical differences in single factor analysis were used as independent variables for multiple stepwise regression analysis. The independent variables of single factor with statistical difference included department, hospital level, position, professional technical title, working years, academic degree and had received diabetes specialist training. The independent variable assignment method is shown in Table 
4; multiple regression analysis shows that the nurse's department and whether he has received relevant training in diabetes are important factors influencing the clinical nurse's blood glucose monitoring KAP score $(\mathrm{P}<0.05)$, and the multiple regression analysis results are shown in Table 5.

Table 3. Five entries with the lowest scores for blood glucose monitoring knowledge, attitude, behavior $(\mathrm{N}=134)$.

\begin{tabular}{|c|c|c|}
\hline Entry & $\begin{array}{c}\text { Average } \chi \pm S \\
\text { of entries }\end{array}$ & $\begin{array}{l}\text { Score } \\
\text { Rate }\end{array}$ \\
\hline \multicolumn{3}{|l|}{ Knowledge } \\
\hline Differences in blood glucose values A4. different blood samples & $3.25 \pm 0.68$ & $65.07 \%$ \\
\hline Blood glucose control targets A6. different populations & $3.36 \pm 0.77$ & $67.16 \%$ \\
\hline $\begin{array}{l}\text { A8. differences between rapid blood glucose monitoring and } \\
\text { biochemical results }\end{array}$ & $3.19 \pm 0.76$ & $63.88 \%$ \\
\hline Effects of A10. hematocrit on blood glucose outcomes & $3.08 \pm 0.86$ & $61.49 \%$ \\
\hline Effect of A11. oxygen partial pressure on blood glucose outcome & $3.02 \pm 0.82$ & $60.29 \%$ \\
\hline \multicolumn{3}{|l|}{ Attitude } \\
\hline Wash hands before B1. blood sugar test & $4.10 \pm 0.82$ & $82.08 \%$ \\
\hline B2. check proper storage of test strips and quality control products & $4.24 \pm 0.72$ & $84.77 \%$ \\
\hline B4. regular blood sugar cleaning & $4.16 \pm 0.77$ & $83.28 \%$ \\
\hline B6. selection of blood collection sites & $4.21 \pm 0.71$ & $84.17 \%$ \\
\hline B7. until alcohol is dry before skin puncture & $4.27 \pm 0.71$ & $85.37 \%$ \\
\hline \multicolumn{3}{|l|}{ Conduct } \\
\hline C2. alcohol to disinfect skin and collect blood & $3.24 \pm 1.51$ & $64.77 \%$ \\
\hline C3. Squeezing Blood Collection & $3.57 \pm 1.09$ & $71.34 \%$ \\
\hline C5. weekly blood sugar meter quality control (with quality control liquid) & $3.43 \pm 1.19$ & $68.65 \%$ \\
\hline C6. blood sugar meter and biochemical comparison every six months & $3.34 \pm 1.22$ & $66.86 \%$ \\
\hline $\begin{array}{l}\text { C13. repeated monitoring is still low blood sugar waiting for doctor's } \\
\text { advice before processing }\end{array}$ & $3.57 \pm 1.14$ & $71.34 \%$ \\
\hline
\end{tabular}

Table 4. Independent variable assignment method of KAP influencing factors for blood glucose monitoring.

\begin{tabular}{|c|c|}
\hline Independent variables & Assignment method \\
\hline Departments & $\begin{array}{c}\text { Internal }=1, \text { Surgical }=2, \text { Obstetrics }=3, \text { Pediatrics }=4, \\
\text { Intensive Medicine }=5, \text { Emergency }=6, \text { Other }=7\end{array}$ \\
\hline Hospital level & Level 1 hospital $=1$, level 2 hospital $=2$, level 3 hospital $=3$ \\
\hline Positions & $\begin{array}{c}\text { Nurse }=1, \text { Nursing Leader }=2 \text {, Head Nurse }=3, \\
\text { Nursing Manager }=4\end{array}$ \\
\hline Professional technical title & Nurse $=1$, Nurse $=2$, Head Nurse $=3$ \\
\hline Number of years worked & $\begin{array}{c}1-5 \text { years }=1,6-10 \text { years }=2,11-20 \text { years }=3, \\
21-30 \text { years }=4,31 \text { years and above } 5\end{array}$ \\
\hline Education & Technical secondary $=1$, junior college $=2$, undergraduate $=3$ \\
\hline $\begin{array}{c}\text { Have you received diabetes } \\
\text { specialist training }\end{array}$ & No $=0$, yes $=1$ \\
\hline
\end{tabular}


Table 5. KAP multiple stepwise regression analysis of blood glucose monitoring $(\mathrm{N}=$ 134).

\begin{tabular}{cccccc}
\hline Independent variables & B & SE & Beta & t & p \\
Constant & 304.526 & 28.923 & - & 10.529 & 0.000 \\
Departments & -4.168 & 2.053 & -0.175 & -2.03 & 0.044 \\
Hospital level & -18.091 & 9.771 & -0.162 & -1.852 & 0.066 \\
Positions & 0.148 & 2.914 & 0.005 & 0.051 & 0.96 \\
Professional technical title & 5.004 & 4.857 & 0.099 & 1.03 & 0.305 \\
Number of years worked & 2.06 & 1.881 & 0.097 & 1.095 & 0.275 \\
Education & 2.977 & 5.815 & 0.045 & 0.512 & 0.61 \\
$\begin{array}{c}\text { Have you received diabetes } \\
\text { specialist training }\end{array}$ & 21.379 & 9.057 & 0.202 & 2.36 & 0.020 \\
\hline
\end{tabular}

Note: $\mathrm{R}^{2}=0.127$, adjusted $\mathrm{R}^{2}=0.078, \mathrm{~F}=2.609, \mathrm{P}=0.015$.

\section{Discussion}

\subsection{Analysis of the Level of Knowledge and Belief in Blood Glucose Monitoring and Management of Clinical Nurses in Nyingchi Region, Tibet}

1) The knowledge of blood glucose monitoring management is at a medium level. The results of this study show that the knowledge level of blood glucose monitoring management is at a medium level, the knowledge score rate is $66.8 \%$, and the total knowledge average score is $(36.78 \pm 6.80)$ points, which is lower than that of clinical nurses in Grade A hospitals in Guangdong Province [7]. The reasons for this analysis were that only $13.4 \%$ of the nurses surveyed had received specialized training in diabetes, while $86.6 \%$ of the nurses did not, and the average knowledge score of the nurses who had participated in the training was higher than that of the nurses who had not participated in the training, indicating that the nurses who had received specialized training in diabetes had a relatively good level of knowledge. The entry with the lowest score in the knowledge dimension is "the effect of oxygen partial pressure on blood glucose outcomes". High altitude hypoxia environment, oxygen partial pressure, disinfectants, drugs and so on will affect the results of blood glucose monitoring [8] [9] [10] [11] [12]; in addition, the Qinghai-Tibet Plateau has a special geographical environment, low oxygen content and oxygen content in the atmosphere, hypoxia causes metabolic disorders of lipids and mucopolysaccharide, and increases the incidence of diabetes [13]. Studies have shown that the vast majority of nurses in Tibet still do not have the opportunity to continue their education for life [14]. The lack of training opportunities for clinical nurses in Nyingchi area of Tibet has led to a low rate of knowledge on blood glucose monitoring and management. Therefore, nurses in Nyingchi area of Tibet, especially nurses in primary hospitals, should be taken as the key object to train the knowledge of blood glucose monitoring and management, so as to strengthen the professional know- 
ledge level of clinical nurses.

2) The attitude of blood glucose monitoring and management is more positive. This study showed that the attitude of clinical nurses in Nyingchi region of Tibet was positive, with a score rate of $84.95 \%$, and the total attitude score was $(42.48 \pm 6.42)$, which was still lower than that of clinical nurses in Grade $3 \mathrm{~A}$ Hospital of Guangdong Province [7]. The lowest score in the attitude dimension was "hand washing before blood glucose testing", with Liu Xueyan et al. [7]. The results are the same, according to Qin Yao et al. [15]. The results of the study showed that there were several reasons: a) the patients did not know enough; b) the medical staff did not know enough; and c) the blood glucose meter was used to explain the defects. Before collecting blood at your fingertips, wash and dry your hands thoroughly, and then tie the first drop of blood in your fingertips, according to organizations such as the American Diabetes Association [16]. Therefore, it is very important to do a good job of correct education and guidance for patients and their families, strengthen the training of nursing staff, and call on manufacturers of blood sugar meters to attach great importance to the instructions.

3) The practice of blood glucose monitoring is not ideal. The results of this study showed that the score rate of blood glucose monitoring management in clinical nurses in Linzhi region of Tibet was 77.33\%, and the total behavior score was $(61.87 \pm 10.24)$. Table 3 shows that the scores of "blood glucose meter and biochemical contrast every six months" and "weekly blood glucose meter quality control (with quality control solution)" were only $66.86 \%$ and $68.65 \%$, respectively. Most nurses cannot make regular biochemical comparison and quality management of blood sugar meter. The study shows that the rapid blood sugar meter should be compared with the biochemical analyzer to improve the accuracy of the rapid blood sugar meter [17]. Guo Xiaotong et al. [18] proposed that routine quality control can ensure the accuracy of blood glucose test results, but most medical institutions do not regularly carry out indoor/room quality control.

\subsection{Analysis of Influencing Factors of Blood Glucose Monitoring KAP of Clinical Nurses in Nyingchi, Tibet}

Table 5 shows that the regression coefficient values of hospitals, titles, titles, years of work, and academic qualifications $(\mathrm{B})>\mathrm{t}$-values, and P-values are all $>$ 0.05 , meaning that there will be no relationship with the total score; $(t=-2.030$, $\mathrm{P}=0.044<0.05$ ), which means that the department will have a significant negative impact on the total score of KAP; whether there is a difference in the blood glucose monitoring management score of the nurses in the endocrinology department. Whether the regression coefficient value of diabetes training has been $21.379(\mathrm{t}=2.360, \mathrm{P}=0.020<0.05)$ means that it will have a significant positive impact on the total KAP score. Whether or not to participate in diabetes specialist training is related to knowledge, belief and behavior scores, and the higher 
the scores of each dimension of clinical nurses trained. The department of nurses and whether they have received diabetes specialist training is an important influencing factor of blood glucose monitoring in clinical nurses in Nyingchi region of Tibet. In addition, this survey shows that the number of people who have participated in diabetes training is only $13.43 \%$, suggesting that the current diabetes nursing training work of clinical nurses in Nyingchi area of Tibet still needs to be strengthened, so nursing managers should strengthen the continuing education training of nurses, establish the training management system of diabetes specialist nurses and contact nurses, and provide more training opportunities in and out of the hospital.

\subsection{Limitations of the Study}

The Knowledge-attitude/belief-practice questionnaire designed for clinical nurses in the Nyingchi area of Tibet in this study cannot contain all the knowledge, attitudes, and behaviors related to clinical nurses' blood glucose monitoring. The questionnaire still has the value of further improvement, making it possible. The questionnaire scale applied to the survey of knowledge, attitude and behavior of blood glucose monitoring of clinical nurses in different areas. In addition, the study only investigates the knowledge and practice of blood glucose monitoring of clinical nurses in Nyingchi, Tibet. The amount of data collected is limited by regional factors and can only partially reflect the knowledge, attitudes, and behaviors of clinical nurses in Tibet on blood glucose monitoring management. In our research, we still need to expand the sample size collected and deepen our understanding of the clinical nurses "blood glucose monitoring and management behaviors in Nyingchi, Tibet from multiple dimensions to facilitate the development of guidance and guidance for nurses" blood glucose monitoring and management in Nyingchi, Tibet.

\section{Conclusion}

The high-calorie, high-protein, high-salt, high-fat and high-sugar structure of the local population in Nyingchi, Tibet, and the habit of drinking alcohol for a long time have led to an increasing incidence of diabetes in the region. The low oxygen environment there will lead to a strong, sticky and concentrated blood plateau characteristics, which will easily aggravate the symptoms of hypoxia and ischemia in patients with diabetes, and promote the development of diabetes [19] [20]. In the course of this survey on the current situation of clinical nurses in Nyingchi region, Tibet, they found that the education level of regional nurses was relatively low, and the opportunities for nurses to go out for further education and training were relatively small. In this survey, the clinical nurses' knowledge and cognition of blood glucose monitoring and management was at the middle level, the attitude was positive, and the behavior of blood glucose monitoring and management was not ideal. It is suggested that nursing managers should conduct standardized training for clinical nurses in blood glucose moni- 
toring, develop diabetes specialist nursing talents in Nyingchi area, and construct a complete diabetes nursing management system.

\section{Funding Project}

Diabetes intelligent wearable equipment and complication prevention and control cloud platform, 2016 Guangdong province science and technology development project number: 2016 B010108008.

\section{Conflicts of Interest}

The authors declare no conflicts of interest regarding the publication of this paper.

\section{References}

[1] Huang, L.J., Lu, L., Fang, M. and Lei, Y.X. (2020) Advances in TCM Dialectical Nursing for Type 2 Diabetes Mellitus. General Practice Care, 18, 540-542.

[2] Yang, W.Y., Lu, J.P., et al. (2010) Prevalence of Diabetes among Men and Women in China. The New England Journal of Medicine, 362, 1090-1101.

[3] Li, X.W. and Lu, Y. (2014) Current Situation and Some Suggestions of Diabetes Health Education in Plateau Area. Journal of Highland Medicine, 14, 56-57.

[4] Dai, Q.X. (2009) Prevention and Treatment of Diabetic Complications in Plateau Region. Qinghai Medical Journal, 39, 76-77.

[5] Zhong, Y. and Qiu, C.Q. (2017) Tang Province Three, Wang Na. A Study on the Effect of WeChat Platform Training on Blood Glucose Monitoring in New Nurses. Journal of Applied Clinical Nursing, 2, 161-162.

[6] American Diabetes Association (2018) Standards of Medical Care in Diabetes 2018. Diabetes Care, 41, 1-159.

[7] Zhou, P.R., Liu, X.Y., Huang, J.W. and Chen, W.J. (2012) Investigation on the Management of Blood Glucose Monitoring by Clinical Nurses of Grade 3A Hospital in Guangdong Province. Journal of Nursing Administration, 12, 466-468.

[8] Hao, K. (2014) CRHR1 Regulatory Mechanisms of Inhibition of Insulin Secretion by High Altitude Hypoxia. Zhejiang University, Hangzhou.

[9] Ding, W. (2019) Effects of Two Skin Disinfection Methods on Peripheral Blood Glucose Monitoring in Diabetic Patients. New Diabetes Mellitus, 22, 3-5.

[10] Zhang, Y., Song, J.J., Gao, S.Q. and Zhao, R.R. (2018) Analysis on the Status of Peripheral Blood Glucose Monitoring. Journal of Binzhou Medical College, 41, $292-293+297$.

[11] Guan, X.H., Zhou, P.R., Huang, J.W., et al. (2010) Analysis of the Problems of Blood Glucose Monitoring in Outpatients. Journal of Nursing Studies, 25, 166-167.

[12] Yu, Z.X., Gu, Q., Liu, N., et al. (2010) A study on the Influencing Factors of Blood Glucose Monitoring by Continuous Venous Blood Filtration in Critically Ill Patients. Chinese Journal of Respiratory and Critical Care, 9, 85-87.

[13] Lei, H. (2013) Correlation Analysis of High Blood Lipid and Carotid Atherosclerotic Plaque Formation in Middle-Aged People in Plateau Area. Qinghai Medicine, 43, 22-23.

[14] Liu, M. (2002) Analysis and Countermeasures of Nurses' Continuing Education. Journal of Tibetan Medicine, No. 3, 34-36. 
[15] Qin, Y. and Dai, H. (2014) Investigation and Analysis of Hand Washing before Blood Glucose Test by Fingertip Blood Collection. Nursing Practice and Research, 11, 104-105.

[16] Diabetes Net. Dutch Study on the Use of Second Drops of Blood in Blood Glucose Testing without Hand Washing. http://www.tnbz.com/a/redian/2011/3303.html

[17] Sun, B. (2018) Comparative Analysis of Blood Glucose Results by Rapid Blood Glucose Meter and Biochemical Analyzer. China Medical Device Information, 24, 8-9.

[18] Guo, X.T., Liu, P.Y. and Ma, H.M. (2020) Instant Detection-Status of Use and Management of Portable Blood Glucometer. Tianjin Nursing, 28, 123-125.

[19] Zhang, P. (2015) Analysis of the Correlation between Microangiopathy and Hematology in Patients with Type 2 Diabetes in Plateau Region. Chinese Experimental Diagnostics, 19, 1510-1511.

[20] Chaido (2016) Analysis of Clinical Characteristics of Tibetan Patients with Different Age Diabetes Mellitus and Pneumonia in Plateau Region. Journal of Nantong University: Medical Edition, 36, 300-302. 